\title{
Caracterização de Phantoms usando Sonda Coaxial para Aplicações Biomédicas
}

\author{
Joanderson Marinho, Erik F. Silva, Kesia C. Santos
}

Resumo-A utilização da técnica de imagens médicas usando as faixas de frequências de micro-ondas para diagnóstico de algumas anormalidades no corpo é tópico de grande interesse, diversos grupos de pesquisa ao redor do mundo têm concentrado esforços nesta linha de pesquisa. Uma parte preliminar e essencial à realização dos testes clínicos é a experimentação em phantoms ou tecidos animais ex-vivo. Este trabalho apresenta um material fabricado com características dielétricas semelhantes àquelas do tecido adiposo e sangue humanos. Os materiais usados e o processo de fabricação são simples. Os resultados foram obtidos usando uma sonda coaxial e apresentam boa concordância com os valores esperados.

Palavras-Chave- Propriedades dielétricas, sonda coaxial, phantom de tecidos biológicos, medidas bioeletromagnéticas.

\section{INTRODUÇÃO}

Nos últimos anos foi crescente o interesse no desenvolvimento de tecnologias de imagens médicas usando frequências na faixa de micro-ondas. Os avanços alcançados nas pesquisas apresentam resultados promissores, especificamente para a detecção precoce de câncer de mama [1], [2]. Na detecção mais rápida de AVC (Acidente Vascular Cerebral) [3]. Em análises ósseas e de cartilagens [4].

A interação das ondas eletromagnéticas com o corpo humano é diretamente dependente das propriedades dielétricas dos tecidos. Antes da técnica poder ser aplicada no corpo humano ou animal são necessários testes para aperfeiçoar o conhecimento. Estes testes são desenvolvidos usando phantoms, que são materiais criados para simular adequadamente as propriedades dielétricas (permissividade e condutividade) do corpo humano ou animal, ou seja, modelos equivalentes aos tecidos biológicos [5], [6].

Atualmente, vários grupos de pesquisa têm desenvolvido phantoms nas formas líquidas, gelatinosas e semi-sólidas, usando modelos 3D impressos [7] para testes em várias aplicações biomédicas. A escolha do tipo de material que será utilizado deve levar em consideração algumas questões, tais como, disponibilidade de materiais, processo de fabricação, e conservação das propriedades dielétricas a longo prazo.

Neste trabalho optou-se por usar mateias líquidos para construir o phantom. A receita usa Triton X- 100 (TX-100), água destilada e sal. Uma vez que os materiais para a fabricação são de fácil aquisição, o processo de fabricação é simples e o tempo de conservação das propriedades dielétricas do phantom é satisfatório [6]. A principal questão observada é evitar a desidratação da mistura criada.

Joanderson Marinho, IFPB, Erik F. Silva, IFPB, Kesia C. Santos, , IFPB, João Pessoa-PB, e-mail: kesia.farias@iffpb.edu.br. Este trabalho foi parcialmente financiado por Interconecta do IFPB).
Após a fabricação dos phantoms foi utilizada uma sonda coaxial Keysight $85070 \mathrm{E}$ disponível no laboratório do grupo [8]. A validação das propriedades dielétricas medidas nos phantoms fabricados é feita comparando os resultados provenientes da medição com os dados de referência existentes e amplamente aceitos [9].

O trabalho tem como objetivo obter experiência na fabricação dos phantoms que podem ser usados numa etapa futura para estudo de anomalias no corpo humano. Assim, com base em receitas já existentes, o tecido mamário saudável (tecido adiposo) e o sangue foram fabricados. As propriedades dielétricas foram analisadas para uma faixa ampla de frequências de 2-14 GHz.

\section{MATERIAIS E MÉTODOS EXPERIMENTAIS}

Em laboratório foi fabricado um phantom que consiste na mistura de materiais de baixo custo e fácil aquisicão: TX -100, água destilada e sal $(\mathrm{NaCl})$, na proporção das receitas sugeridas em [5] para o tecido adiposo e em [6] para o sangue, ambas para a faixa de 1-6 GHz. Os resultados da receita foram validados para uma faixa mais ampla de 2-14 GHz. A adição de sal à mistura tem por objetivo controlar a condutividade. A Tabela I apresenta a receita usada.

TABELA I. RECEITA DOS LÍQUIDOS PARA OS PHANTOMS.

\begin{tabular}{|c|c|c|c|}
\hline Tecido & $\begin{array}{c}\text { TX-100 } \\
(\text { vol \%) }\end{array}$ & $\begin{array}{c}\text { Água destilada } \\
\text { (vol \%) }\end{array}$ & $\mathrm{NaCl}$ \\
\hline $\begin{array}{c}\text { Tecido } \\
\text { adiposo }\end{array}$ & 100 & 0 & 0 \\
\hline Sangue & 20 & 80 & $9 \mathrm{~g} / \mathrm{L}$ \\
\hline
\end{tabular}

Para a amostra de sangue foi adicionada metade da quantidade do TX - 100 ao recipiente, em seguida o volume completo da solução água destilada-sal, o composto precisa ser vigorosamente misturado até obter aparência homogênea. O último passo é adicionar a parte restante do TX -100 e novamente realizar a mistura dos líquidos. Este procedimento de fabricação evita a formação de bolhas de ar na mistura e garante a homogeneidade entre os dois líquidos misturados.

Após este procedimento, os líquidos fabricados estão prontos para a etapa da medição. Nesta etapa, conferimos se os valores das propriedades dielétricas dos phantoms criados correspondem aos valores esperados para os tecidos biológicos demonstrados pelo modelo de Cole-Cole [9].

A sonda coaxial Keysight 85070 E foi usada para medir as propriedades dielétricas do phantom na faixa de frequências de 2-14 GHz. As amostras foram medidas à temperatura ambiente, o procedimento de calibração da sonda foi realizado 
adequadamente: em aberto, em curto e com o líquido padrão que no nosso caso foi água destilada [10].

Para as medições das amostras foi utilizado um analisador vetorial de rede conectado à sonda coaxial para coleta dos dados, conforme ilustrado na Figura 1.

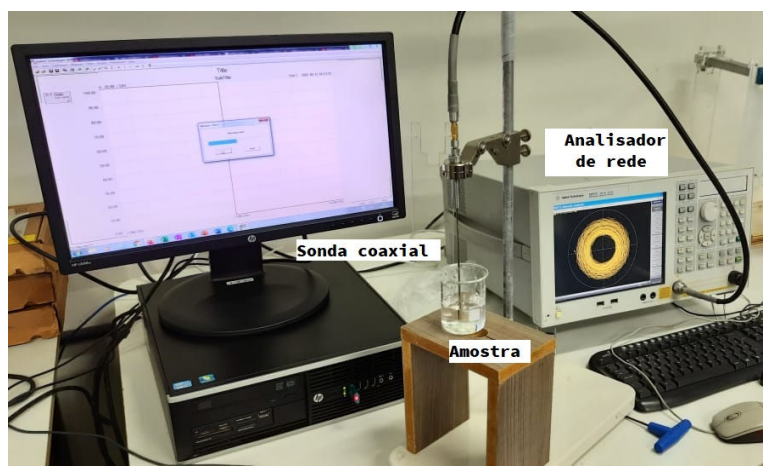

Fig. 1 Setup de medição do phantom utilizando a sonda coaxial.

As características dielétricas (permissividade relativa e condutividade) para os phantoms fabricados são comparadas com o modelo de Cole-Cole para propriedades dielétricas.

$$
\varepsilon^{*}=\varepsilon_{\infty}+\sum_{n} \frac{\Delta \varepsilon_{n}}{1+\left(j \omega \tau_{n}\right)^{1-\alpha_{n}}}+\frac{\sigma}{j \omega \varepsilon_{0}}
$$

em que $\omega$ é a frequência angular, $\sigma$ é a condutividade, $\tau$ is the tempo de relaxação, $\mathrm{n}$ é o número de termos, $\varepsilon_{0}$ é a permissividade do espaço livre, $\Delta \varepsilon_{\mathrm{n}}=\varepsilon_{\mathrm{s}, \mathrm{n}}-\varepsilon_{\infty}$, em que $\varepsilon_{\mathrm{s}, \mathrm{n}}$, e $\varepsilon_{\infty}$ são as constantes dielétricas estática e infinita, respectivamente. A Tabela II apresenta os valores usados no modelo de Cole-Cole. Os resultados são apresentados na Figura 2.

\section{TABELA II. PARAMETROS DO MODELO DE COLE-COLE DO PHANTOM.}

\begin{tabular}{|c|c|c|c|c|c|}
\hline Tecido & $\varepsilon_{\infty}$ & $\Delta \varepsilon_{1}$ & $\begin{array}{c}\tau_{1} \\
(\mathrm{ps})\end{array}$ & $\alpha_{1}$ & $\sigma(\mathrm{S} / \mathrm{m})$ \\
\hline $\begin{array}{c}\text { Tecido } \\
\text { adiposo }\end{array}$ & 2.2 & 3 & 7.96 & 0.2 & 0.01 \\
\hline Sangue & 4 & 40 & 8.38 & 0.1 & 0.7 \\
\hline
\end{tabular}

Observa-se uma boa concordância entre os valores medidos e os esperados pelo modelo de Cole-Cole para os phantoms fabricados em nosso laboratório. Esta concordância não é observada em toda a faixa de frequência, o que nos aponta que a receita usada pode ser alterada buscando esta concordância para estas faixas. No entanto, é válido assumir que o tecido mamário saudável e o sangue podem ser representados por estes líquidos fabricados, na faixa de $2-14 \mathrm{GHz}$.

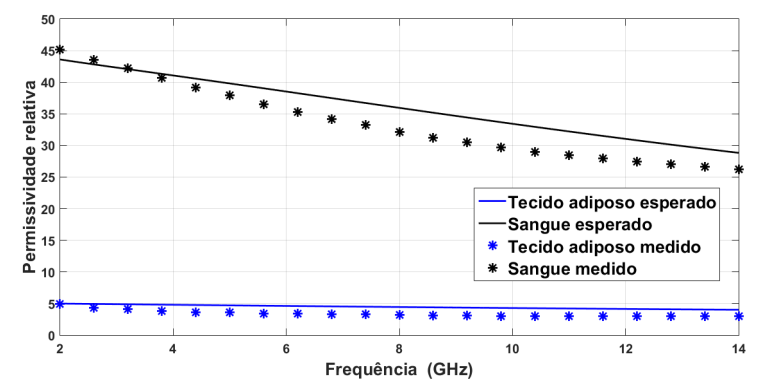

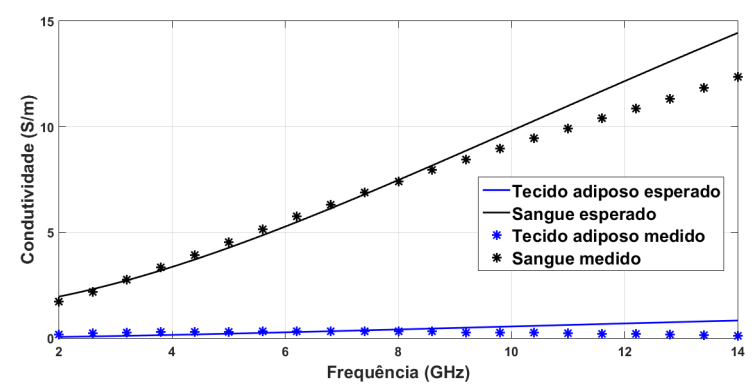

Fig. 2 Propriedades dielétricas dos phantoms: (a) permissividade relativa, (b) condutividade.

\section{CONCLUSÕES}

A caracterização dielétrica de um phantom foi realizada com boa concordância entre os valores medidos e os valores esperados. As receitas testadas com base em receitas existentes apresentaram resultados que indicam que elas podem ser extendidas para uma faixa mais ampla de frequências $(2-14$ $\mathrm{GHz}$ ). O processo de fabriação e medição foi executado de forma adequada em laboratório pelos membros da equipe.

Estes phantoms líquidos farão parte do banco de materiais desenvolvidos em nosso laboratório, o nosso objetivo principal foi a fabricação e caracterização dielétrica de materiais que simulam as propriedades dielétricas de tecidos biológicos.

Em estudos futuros, os resultados obtidos neste trabalho podem contribuir para a análise das características dielétricas em tecidos animais ex-vivo. Além de proporcionar experiência para a caracterização dos tecidos biológicos com a sonda coaxial.

\section{REFERÊNCIAS}

[1] E. C. Fear e a. M. A. Stuchly, "Microwave detection of breast cancer," IEEE Transactions on Microwave Theory and Techniques, vol. 48, $\mathrm{n}^{\circ} 11$, pp. 1854-1863, 2000.

[2] E. Fear, S. Hagness, P. Meaney, M. Okoniewski e M. Stuchly, "Enhancing breast tumor detection with near-field imaging," IEEE Microwave Magazine, vol. 3, pp. 48 - 56, August 2002.

[3] B. J. Mohammed, A. M. Abbosh, S. Mustafa e D. Ireland, "Microwave System for Head Imaging," IEEE Transactions on Instrumentation and Measurement, vol. 63, pp. 117 - 123, August 2013.

[4] R. Chandra, H. Zhou, I. Balasingham e R. M. Narayanan, "On the Opportunities and Challenges in Microwave Medical Sensing and Imaging," IEEE Transactions on Biomedical Engeneering, vol. 62, pp. 1667-1681, 2015.

[5] N. Joachimowicz, C. Conessa, T. Henriksson e B. Duchêne, "Breast Phantoms for Microwave Imaging," IEEE Antennas and Wireless Propagation Letters, vol. 13, 2014.

[6] N. Joachimowicz, B. D. C. Conessa1 e O. Meyer, "Reference Phantoms for Microwave Imaging," em 2017 11th European Conference on Antennas and Propagation (EUCAP), 2017.

[7] A. T. Mobashsher e A. M. Abbosh, "Artificial Human Phantoms," IEEE Microwave Magazine, pp. 42-62, July 2015.

[8] Keysight, "85070E Dielectric Probe Kit $200 \mathrm{MHz}$ to $50 \mathrm{GHz}$ : Technical Overview," 2017.

[9] S. Gabriely, R. W. Lau e a. C. Gabriel, "The dielectric properties of biological tissues III. Parametric models for the dielectric spectrum of tissues," Phys. Med. Biol., vol. 41, 1996.

[10] A. L. Gioia, E. Porter, I. Merunka, A. Shahzad, S. Salahuddin, M. Jones e a. M. O'Halloran, "Open-Ended Coaxial Probe Technique for Dielectric Measurement of Biological Tissues: Challenges and Common Practices," Diagnostics, vol. 8, pp. 1-38, 2018. 